brought up by reputable third-party information sources. All requests for action are first cross-checked through the Clearinghouse on Science and Human Rights of the American Association for the Advancement of Science.

The Ethics Committee will respond to cases of human rights violations involving scholars whose field correspond to those subsumed under the phrase "political science" in the United States. It will also take up cases that do not directly involve political scientists but have broad implications for all social sciences.

Given the limited time and resources of the Ethics Committee, it will consider only what appear to be the most egregious cases of human rights violations. The standard being used in the making of this choice will be the International Declaration of Human Rights and the two accompanying covenants. As a general rule the Committee will take up no more than six cases at a time.

The Ethics Committee has already worked on behalf of political scientists in Ethiopia, the Philippines, Yugoslavia, Turkey, the People's Republic of China, Guatemala, Nicaragua, and South Africa. The procedure the Committee follows is to write letters of inquiry to the appropriate authorities and to follow up this letter with subsequent letters, if necessary. Other activities such as visits to embassies and site visits can also be considered by the Committee.

Members of the Association having in- formation pertaining to human rights abuses are urged to contact the Ethics Committee.

\section{Report of the Treasurer of the American Political Science Association, 1988-89}

\author{
Nancy H. Zingale \\ College of St. Thomas
}

The Association ended 1988-89 with a budget surplus of $\$ 115,901$, the tenth straight year in which a surplus was recorded. Revenue increased by 12.8 percent over the prior year while expenditures increased by 10.7 percent (see Table 1). The relatively large increases in both income and spending were due in part to the inclusion in this year's budget of the 1988 meeting of the International Political Science Association, held in Washington in conjunction with the APSA's annual meeting. While the APSA's hosting of the IPSA meeting generated additional revenues as well as expenses, this obligation represented a net loss for the Association of $\$ 33,118$, making the overall surplus and increase in income relative to spending all the more impressive. The Association's

Table 1. APSA Budget: A Ten-Year Perspective

\begin{tabular}{ccccc}
\hline \hline Year & Income & Expenditures & $\begin{array}{c}\text { Surplus }+ \\
\text { Deficit }-\end{array}$ & $\begin{array}{c}\text { Annual } \\
\text { Expenditure } \\
\text { Change (\%) }\end{array}$ \\
\hline $1979-80$ & $\$ 930,157$ & $\$ 929,857$ & +300 & +6.2 \\
$1980-81$ & $1,007,675$ & 977,328 & $+30,347$ & +5.1 \\
$1981-82$ & $1,117,701$ & $1,043,255$ & $+74,446$ & +6.7 \\
$1982-83$ & $1,202,078$ & $1,094,415$ & $+107,663$ & +4.9 \\
$1983-84$ & $1,323,074$ & $1,247,529$ & $+75,545$ & +14.0 \\
$1984-85$ & $1,413,078$ & $1,353,339$ & $+59,739$ & +8.5 \\
$1985-86$ & $1,505,224$ & $1,453,248$ & $+51,976$ & +6.9 \\
$1986-87$ & $1,584,945$ & $1,499,638$ & $+85,307$ & +3.1 \\
$1987-88$ & $1,637,637$ & $1,563,252$ & $+74,385$ & +4.2 \\
$1988-89$ & $1,847,152$ & $1,731,251$ & $+115,901$ & +10.7 \\
\hline
\end{tabular}


Table 2. Revenue Trends, 1984-89

\begin{tabular}{lrrrrr}
\hline \hline & $1984-85$ & $1985-86$ & $1986-87$ & $1987-88$ & $1988-89$ \\
\hline Individual Membership & $\$ 390,044$ & $\$ 398,408$ & $\$ 435,393$ & $\$ 432,902$ & $\$ 488,721$ \\
Institutional Membership & 252,138 & 314,745 & 291,120 & 301,359 & 303,809 \\
Grants (Overhead) & 94,363 & 102,841 & 101,709 & 89,599 & 74,514 \\
Annual Meeting & 83,585 & 72,610 & 89,610 & 87,565 & 134,351 \\
Advertising & 175,883 & 201,138 & 217,880 & 217,431 & 256,164 \\
Dividends/Interest & 118,840 & 119,393 & 146,884 & 191,157 & 184,872 \\
Sales & 67,102 & 53,143 & 53,959 & 59,172 & 74,853 \\
Departmental Services & 102,588 & 97,175 & 94,849 & 91,382 & 145,281 \\
Other & 128,535 & 145,771 & 153,542 & 167,070 & 184,587 \\
TOTAL & $\$ 1,413,078$ & $\$ 1,505,224$ & $\$ 1,584,945$ & $\$ 1,637,637$ & $\$ 1,847,152$ \\
\hline
\end{tabular}

healthy financial situation stems from prudent management by Executive Director Catherine Rudder and from a number of revenue raising strategies adopted by the Council and imaginatively designed and effectively implemented by the Association's excellent staff.

\section{Revenue Trends}

During the last two years, the Council approved the investment of resources for improving the recruitment and retention of members, for increasing the sale of advertising in its publications, and for increasing participation in the Departmental Services Program. These strategies have yielded significant results. Income from individual memberships rose 13 percent, advertising revenues increased 18 percent and income from the Departmental Services Program increased 59 percent (see Table 2). Included in the increased individual memberships is $\$ 30,000$ in life memberships, a result of the Council's action to increase life membership dues to $\$ 2,000$, but offer such memberships for $\$ 1,000$ if paid in full by December 31 , 1988. (Although included in the 1988-89 revenue figures, this $\$ 30,000$ has been transferred to the Trust and Development Fund.) Revenue from the annual meeting increased 53 percent, due in substantial part to larger attendance and an increased number of exhibitors generated by the IPSA meeting. The publication of a new biographical directory produced an increase in sales revenue. Although revenue from grants declined due to the phasing out of Project ' 87 , its continuance into the current year provided more overhead revenue than had been originally budgeted. These increases more than offset a slight decline of 3 percent in dividend and interest income.

\section{Spending Trends}

The strategies that produced the increased revenues required increased expenditures during 1988-89 (see Table 3). The costs of maintaining the membership, business office expenses and sales expenses increased by 17 percent, yet only returned to the level of expenditures in the previous year. Expenses associated with the IPSA appear as a separate entry in Table 3 as well as in added costs for the annual meeting. The cost of publication of the biographical directory accounts for the increase in expenditures for special programs. A reduction in general operating expenses reflects the careful management practices of the APSA staff.

\section{Budget Actions of the Council}

The Council has taken a number of actions to keep the Association on a sound financial footing. The transfer of $\$ 30,000$ from the 1988-89 operating budget to the Trust and Development Fund was authorized in order to amortize the life memberships acquired during that year. A differential dues increase for the Departmental 
Table 3. Expenditure Trends, 1984-89

\begin{tabular}{|c|c|c|c|c|c|}
\hline & $1984-85$ & $1985-86$ & $1986-87$ & $1987-88$ & $1988-89$ \\
\hline $\begin{array}{l}\text { Publications } \\
\text { Annual Meeting } \\
\text { Special Programs } \\
\text { Governing the }\end{array}$ & $\begin{array}{r}\$ 276,174 \\
85,063 \\
224,360\end{array}$ & $\begin{array}{l}\$ 309,251 \\
113,059 \\
234,391\end{array}$ & $\begin{array}{r}\$ 300,030 \\
111,347 \\
229,030\end{array}$ & $\begin{array}{l}\$ 331,685 \\
112,573 \\
221,291\end{array}$ & $\begin{array}{l}\$ 371,627 \\
125,459 \\
274,492\end{array}$ \\
\hline $\begin{array}{l}\text { Association } \\
\text { Membership, Business }\end{array}$ & 50,518 & 55,932 & 56,721 & 66,212 & 66,366 \\
\hline $\begin{array}{l}\text { Office and Sales } \\
\text { General Operating } \\
\text { IPSA Net Expenses }\end{array}$ & $\begin{array}{l}207,926 \\
509,298\end{array}$ & $\begin{array}{l}204,811 \\
529,126\end{array}$ & $\begin{array}{l}215,565 \\
586,945\end{array}$ & $\begin{array}{l}184,372 \\
647,119\end{array}$ & $\begin{array}{l}215,704 \\
644,485\end{array}$ \\
\hline Over Income & - & - & - & - & 33,118 \\
\hline TOTAL & $\overline{\$ 1,353,339}$ & $\overline{\$ 1,537,318}$ & $\$ 1,499,638$ & $\$ 1,563,252$ & $\$ 1,731,251$ \\
\hline
\end{tabular}

Services Program was authorized in order to more appropriately reflect the usage of such services as advertising in the Personnel Newsletter. A graduated dues schedule for retired members was introduced; retired members with incomes of $\$ 25,000$ or more will pay $\$ 40$ while the dues of those making less than $\$ 25,000$ will remain at $\$ 20$. The Council also took action to gain control over the newsletter expenditures of organized sections that have been underwritten by the Association.

In an effort to insure the viability of the various endowed awards and prizes of the Association, the Council requested that the Board of Trustees of the Trust and Development Fund take responsibility for investing their assets.

At the request of Executive Director Rudder, the Council changed the Association's retirement benefits, authorizing payment of 10 percent of salary into the retirement accounts of all APSA employees. This change represents a decrease in the compensation of professional staff and an increase in that of non-professional employees and was necessitated by new federal regulations prohibiting discrimination among different classes of employees in retirement benefits.

The budget for 1989-90 represents a vigorous effort to contain costs. Nevertheless, a deficit of $\$ 63,206$ has been projected (see Table 5). The largest uncontrollable increase in projected expenses comes from a rapid rise in the price of paper, significantly increasing the costs of publishing the APSR, PS, and The Political Science Teacher. Merit salary increases and a significant rise in health care costs will combine to increase salary expenses by slightly more than 5 percent. A search for a new managing editor for the American Political Science Review, and its associated expenses, have been authorized by the Council. A number of grant opportunities are being pursued which, if successful, will considerably improve the short-term financial picture.

Table 4. APSA Membership, 1984-89

\begin{tabular}{ccccc}
\hline \hline Year & Student & Retired & Total Individual* & $\begin{array}{c}\text { Total Revenue } \\
\text { From Individuals }\end{array}$ \\
\hline $1984-85$ & 2,595 & 411 & 9,273 & $\$ 390,044$ \\
$1985-86$ & 2,589 & 432 & 9,465 & $\$ 398,408$ \\
$1986-87$ & 2,775 & 439 & 9,610 & $\$ 435,393$ \\
$1987-88$ & 2,728 & 450 & 9,837 & $\$ 432,902$ \\
$1988-89$ & 3,054 & 489 & 10,595 & $\$ 488,721$ \\
\hline
\end{tabular}

"Includes associate, life, and family memberships. 
Table 5. Revenue and Expenditures, 1987-90

\begin{tabular}{|c|c|c|c|c|}
\hline & $\begin{array}{r}\text { Actual } \\
1987-88\end{array}$ & $\begin{array}{l}\text { Budget } \\
1988-89\end{array}$ & $\begin{array}{r}\text { Actual } \\
1988-89\end{array}$ & $\begin{array}{r}\text { Budget } \\
1989-90\end{array}$ \\
\hline \multicolumn{5}{|l|}{ REVENUE } \\
\hline $\begin{array}{l}\text { MEMBERSHIP } \\
\text { Professional } \\
\text { Student } \\
\text { Family } \\
\text { Institutional } \\
\text { Life } \\
\text { Associate }\end{array}$ & $\begin{array}{r}376,240 \\
41,274 \\
880 \\
301,359 \\
10,025 \\
4,483\end{array}$ & $\begin{array}{r}386,000 \\
42,000 \\
1,000 \\
301,500 \\
15,000 \\
5,000\end{array}$ & $\begin{array}{r}398,318 \\
49,295 \\
895 \\
303,809 \\
33,010 \\
7,203\end{array}$ & $\begin{array}{r}418,000 \\
52,000 \\
1,000 \\
305,000 \\
2,000 \\
7,500\end{array}$ \\
\hline TOTAL MEMBERSHIP & 734,261 & 750,500 & 792,530 & 785,500 \\
\hline ADMINISTRATIVE & $\overline{89,599}$ & 50,000 & $\overline{74,514}$ & 70,000 \\
\hline ANNUAL MEETING & $\overline{87,565}$ & 95,000 & 134,351 & 107,000 \\
\hline $\begin{array}{l}\text { ADVERTISING } \\
\text { Review } \\
\text { PS } \\
\text { Program } \\
\text { Exhibits } \\
\text { The Political Science Teacher }\end{array}$ & $\begin{array}{r}85,658 \\
13,592 \\
45,108 \\
72,750 \\
323\end{array}$ & $\begin{array}{r}90,000 \\
17,000 \\
55,000 \\
90,000 \\
2,000\end{array}$ & $\begin{array}{r}95,429 \\
9,499 \\
60,155 \\
87,811 \\
3,270\end{array}$ & $\begin{array}{r}101,000 \\
12,000 \\
57,000 \\
85,000 \\
4,000 \\
\end{array}$ \\
\hline TOTAL ADVERTISING & 217,431 & 254,000 & 256,164 & 259,000 \\
\hline DIVIDENDS AND INTEREST & 191,157 & 200,000 & 184,872 & 200,000 \\
\hline $\begin{array}{l}\text { SALES } \\
\text { Back Issues } \\
\text { Mailing Lists } \\
\text { Books, Monographs and Papers } \\
\text { Reprints } \\
\text { State of the Discipline Book } \\
\text { Style Manual }\end{array}$ & $\begin{array}{r}3,146 \\
26,781 \\
18,235 \\
4,740 \\
6,270 \\
-\end{array}$ & $\begin{array}{r}2,000 \\
28,000 \\
19,000 \\
4,000 \\
3,000 \\
\end{array}$ & $\begin{array}{r}3,079 \\
41,093 \\
17,356 \\
4,024 \\
8,242 \\
1,059\end{array}$ & $\begin{array}{r}3,000 \\
45,000 \\
20,000 \\
4,000 \\
4,000 \\
1,500\end{array}$ \\
\hline TOTAL SALES & 59.172 & 56,000 & 74,853 & 77,500 \\
\hline DEPARTMENTAL SERVICES & 91,382 & 145,000 & $\overline{|45,28|}$ & $\overline{142,000}$ \\
\hline RENT & 86,587 & 91,000 & 91,322 & 96,000 \\
\hline ROYALTIES & 25,386 & 25,000 & 16,532 & 15,000 \\
\hline PERSONNEL PLACEMENT SERVICE & 36,378 & 37,000 & 48,636 & 51.000 \\
\hline SECTION DUES & $\overline{17,459}$ & 20,000 & $\overline{26,440}$ & 29,000 \\
\hline MISCELLANEOUS AND CONTRIBUTIONS & 1,260 & 1,500 & 1,657 & 2,000 \\
\hline TOTAL REVENUE & $1,637,637$ & $1,725,000$ & $1,847,152$ & $1,834,000$ \\
\hline \multicolumn{5}{|l|}{ EXPENDITURES } \\
\hline \multicolumn{5}{|l|}{ PUBLICATIONS } \\
\hline $\begin{array}{l}\text { A. REVIEW } \\
\text { Printing } \\
\text { Postage and Mailing } \\
\text { Salaries } \\
\text { Office and Editorial Board Expenses }\end{array}$ & $\begin{array}{r}143,831 \\
21,880 \\
55,171 \\
21,279\end{array}$ & $\begin{array}{r}145,000 \\
25,000 \\
65,000 \\
22,000\end{array}$ & $\begin{array}{r}156,307 \\
26,103 \\
69,834 \\
18,805\end{array}$ & $\begin{array}{r}190,000 \\
28,500 \\
70,200 \\
20,000 \\
\end{array}$ \\
\hline TOTAL REVIEW & 242,161 & 257,000 & 271,049 & $\overline{308,700}$ \\
\hline
\end{tabular}


Table 5 (continued)

\begin{tabular}{|c|c|c|c|c|}
\hline & $\begin{array}{r}\text { Actual } \\
\text { I987-88 }\end{array}$ & $\begin{array}{l}\text { Budget } \\
\text { 1988-89 }\end{array}$ & $\begin{array}{r}\text { Actual } \\
\text { 1988-89 }\end{array}$ & $\begin{array}{r}\text { Budget } \\
1989-90\end{array}$ \\
\hline $\begin{array}{l}\text { B. PS } \\
\text { Printing } \\
\text { Postage and Handling } \\
\text { Editorial Board and Miscellaneous Expenses }\end{array}$ & $\begin{array}{r}57,194 \\
15,520 \\
1,403\end{array}$ & $\begin{array}{r}58,000 \\
17,000 \\
2,000\end{array}$ & $\begin{array}{r}66,598 \\
15,894 \\
1,938\end{array}$ & $\begin{array}{r}80,000 \\
19,380 \\
2,120\end{array}$ \\
\hline TOTAL PS & 74,117 & 77,000 & 84,430 & 101,500 \\
\hline C. THE POLITICAL SCIENCE TEACHER & 15,407 & 17,000 & 16,148 & 18,700 \\
\hline D. OTHER & - & - & - & - \\
\hline TOTAL PUBLICATIONS & 331,685 & 351,000 & 371,627 & 428,900 \\
\hline $\begin{array}{l}\text { ANNUAL MEETING } \\
\text { Printing } \\
\text { Program Committee } \\
\text { General Expenses } \\
\text { Salaries } \\
\text { Exhibits }\end{array}$ & $\begin{array}{r}30,033 \\
6,040 \\
43,055 \\
29,901 \\
3,544\end{array}$ & $\begin{array}{r}32,000 \\
7,500 \\
43,000 \\
40,000 \\
6,000\end{array}$ & $\begin{array}{r}28,557 \\
4,976 \\
51,272 \\
36,210 \\
4,444\end{array}$ & $\begin{array}{r}31,412 \\
6,000 \\
50,000 \\
46,000 \\
6,000\end{array}$ \\
\hline TOTAL ANNUAL MEETING & 112,573 & 128,500 & 125,459 & 139,412 \\
\hline \multicolumn{5}{|l|}{$\begin{array}{l}\text { SPECIAL PROGRAMS } \\
\text { A. APSA COMMITTEE- } \\
\text { TRAVEL AND MEETINGS }\end{array}$} \\
\hline $\begin{array}{l}\text { Status of Blacks } \\
\text { Status of Chicanos } \\
\text { Status of Women } \\
\text { APSR Managing Editor Search Committee } \\
\text { Applied Political Scientists } \\
\text { Education } \\
\text { Ethics, Rights and Freedom } \\
\text { Research Support } \\
\text { Ad Hoc on Structure of Annual Meeting } \\
\text { Publications } \\
\text { International Political Science } \\
\text { Endowed Program Committee }\end{array}$ & $\begin{array}{r}4,511 \\
2,142 \\
2,121 \\
0 \\
58 \\
2,520 \\
4,634 \\
1,987 \\
1,393 \\
1,734 \\
2,808\end{array}$ & $\begin{array}{r}5,000 \\
3,000 \\
3,000 \\
0 \\
0 \\
3,000 \\
5,000 \\
3,000 \\
0 \\
3,000 \\
3,000\end{array}$ & $\begin{array}{r}4,686 \\
1,087 \\
2,395 \\
0 \\
0 \\
2,641 \\
5,092 \\
1,811 \\
0 \\
1,952 \\
3,089\end{array}$ & $\begin{array}{r}5,000 \\
3,000 \\
3,000 \\
3,000 \\
0 \\
3,000 \\
5,000 \\
3,000 \\
0 \\
3,000 \\
3,000\end{array}$ \\
\hline (Development Committee) & 975 & 3,000 & 0 & 3,000 \\
\hline $\begin{array}{l}\text { TOTAL APSA COMMITTEE } \\
\text { TRAVEL AND MEETINGS }\end{array}$ & 24,883 & 31,000 & 22,753 & 34,000 \\
\hline $\begin{array}{l}\text { B. PLACEMENT SERVICE } \\
\text { Printing and Postage } \\
\text { Salaries }\end{array}$ & $\begin{array}{l}22,790 \\
31,227\end{array}$ & $\begin{array}{l}25,000 \\
32,000\end{array}$ & $\begin{array}{l}28,225 \\
26,792\end{array}$ & $\begin{array}{l}31,000 \\
32,000\end{array}$ \\
\hline TOTAL PLACEMENT SERVICE & 54,017 & 57,000 & 55,017 & 63,000 \\
\hline C. BLACK FELLOWSHIP & 12,355 & 18,0010 & 0 & 6,000 \\
\hline D. CHICANO/LATINO FELLOWSHIP & 6,355 & 6,000 & 6,000 & 0 \\
\hline
\end{tabular}


Table 5 (continued)

\begin{tabular}{|c|c|c|c|c|}
\hline & $\begin{array}{r}\text { Actual } \\
1987-88\end{array}$ & $\begin{array}{c}\text { Budget } \\
\text { 1988-89 }\end{array}$ & $\begin{array}{r}\text { Actual } \\
\text { I988-89 }\end{array}$ & $\begin{array}{l}\text { Budget } \\
1989-90\end{array}$ \\
\hline $\begin{array}{l}\text { E. MEMBERSHIP IN OTHER SOCIETIES } \\
\text { COSSA } \\
\text { IPSA } \\
\text { Others }\end{array}$ & $\begin{array}{r}16,000 \\
7,344 \\
10,556\end{array}$ & $\begin{array}{r}16,000 \\
7,344 \\
10,500\end{array}$ & $\begin{array}{r}16,000 \\
7,344 \\
10,863\end{array}$ & $\begin{array}{r}17,000 \\
7,344 \\
11,130\end{array}$ \\
\hline $\begin{array}{l}\text { TOTAL MEMBERSHIP IN } \\
\text { OTHER SOCIETIES }\end{array}$ & 33,900 & 33,844 & 34,207 & 35,474 \\
\hline F. DEPARTMENTAL SERVICES & $\overline{56,405}$ & 105,000 & 126,305 & 96,000 \\
\hline G. EDUCATION PROGRAM & $\overline{7,079}$ & $\overline{15,500}$ & $\overline{1,319}$ & 15,500 \\
\hline H. SECTIONS & 10,764 & 12,000 & 10,674 & 8,000 \\
\hline I. ORAL HISTORY PROJECT & $\overline{1,953}$ & $\overline{2,000}$ & 2,000 & 2,000 \\
\hline J. SMALL GRANTS PROGRAM & 13,580 & 15,000 & 16,217 & 15,000 \\
\hline K. SOUTH AFRICA & 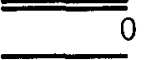 & 5,000 & 0 & 1,000 \\
\hline TOTAL SPECIAL PROGRAMS & 221,291 & 300,344 & $\overrightarrow{274,492}$ & 275,974 \\
\hline $\begin{array}{l}\text { GOVERNING THE ASSOCIATION } \\
\text { Council } \\
\text { Elections } \\
\text { Administrative Committee } \\
\text { Accounting and Legal Fees } \\
\text { Presidential Expenses } \\
\text { Nominating Committee } \\
\text { Elections Committee } \\
\text { Professional Liability Insurance }\end{array}$ & $\begin{array}{r}23,175 \\
5,142 \\
3,523 \\
28,535 \\
3,251 \\
2,105 \\
481 \\
-\end{array}$ & $\begin{array}{r}25,000 \\
5,000 \\
4,000 \\
29,000 \\
4,000 \\
3,000 \\
500 \\
7,000\end{array}$ & $\begin{array}{r}21,287 \\
0 \\
3,115 \\
30,319 \\
4,260 \\
3,022 \\
0 \\
4,363\end{array}$ & $\begin{array}{r}25,000 \\
5,000 \\
4,000 \\
31,000 \\
4,000 \\
3,500 \\
500 \\
8,000\end{array}$ \\
\hline TOTAL GOVERNING THE ASSOCIATION & 66,212 & 77,500 & 66,366 & 81,000 \\
\hline \multicolumn{5}{|l|}{ MEMBERSHIP, BUSINESS OFFICE \& SALES } \\
\hline $\begin{array}{l}\text { MEMBERSHIP RECORDS } \\
\text { Computer Processing \& Membership } \\
\text { Recruitment } \\
\text { Salaries }\end{array}$ & $\begin{array}{r}8,686 \\
80,414 \\
\end{array}$ & $\begin{array}{r}10,000 \\
91,000 \\
\end{array}$ & $\begin{array}{l}16,717 \\
94,301 \\
\end{array}$ & $\begin{array}{r}17,000 \\
101,000 \\
\end{array}$ \\
\hline TOTAL MEMBERSHIP RECORDS & 89,100 & 101,000 & 111,018 & 118,000 \\
\hline $\begin{array}{l}\text { BUSINESS OFFICE } \\
\text { Salaries }\end{array}$ & 53,705 & 63,000 & 62,535 & 69,000 \\
\hline TOTAL BUSINESS OFFICE & 53,705 & 63,000 & 62,535 & 69,000 \\
\hline $\begin{array}{l}\text { COST OF SALES } \\
\text { Printing, Fees, Publicity } \\
\text { Salaries }\end{array}$ & $\begin{array}{l}10,917 \\
30,650\end{array}$ & $\begin{array}{l}11,000 \\
31,000\end{array}$ & $\begin{array}{l}12,607 \\
29,544\end{array}$ & $\begin{array}{l}15,000 \\
33,480\end{array}$ \\
\hline TOTAL COST OF SALES & 41,567 & $\overline{42,000}$ & 42,151 & 48,480 \\
\hline $\begin{array}{l}\text { TOTAL MEMBERSHIP, BUSINESS OFFICE } \\
\& \text { COST OF SALES }\end{array}$ & 184,372 & 206,000 & 215,704 & 235,480 \\
\hline
\end{tabular}


Table 5 (continued)

\begin{tabular}{|c|c|c|c|c|}
\hline & $\begin{array}{l}\text { Actual } \\
1987-88\end{array}$ & $\begin{array}{l}\text { Budget } \\
\text { 1988-89 }\end{array}$ & $\begin{array}{l}\text { Actual } \\
1988-89\end{array}$ & $\begin{array}{l}\text { Budget } \\
1989-90\end{array}$ \\
\hline \multicolumn{5}{|l|}{ GENERAL OPERATING EXPENSES } \\
\hline A. SALARIES & 322,538 & 325,000 & 327,961 & 395,000 \\
\hline $\begin{array}{l}\text { B. BUILDING } \\
\text { Taxes } \\
\text { Repairs and Upkeep } \\
\text { Utilities } \\
\text { Supplies-Building } \\
\text { Insurance-Building } \\
\text { Depreciation } \\
\text { Custodial Salaries }\end{array}$ & $\begin{array}{r}50,750 \\
14,861 \\
22,733 \\
5,708 \\
5,634 \\
12,833 \\
25,556\end{array}$ & $\begin{array}{r}58,300 \\
15,000 \\
24,000 \\
7,000 \\
8,000 \\
12,000 \\
28,000\end{array}$ & $\begin{array}{r}59,175 \\
5,060 \\
24,391 \\
5,484 \\
7,846 \\
12,048 \\
31,445\end{array}$ & $\begin{array}{r}60,000 \\
15,000 \\
25,440 \\
7,000 \\
8,000 \\
12,000 \\
34,000\end{array}$ \\
\hline TOTAL BUILDING & 138,075 & 152,300 & 145,449 & 161,440 \\
\hline $\begin{array}{l}\text { EQUIPMENT, SUPPLIES AND } \\
\text { ADMINISTRATIVE EXPENSES } \\
\text { Postage } \\
\text { Stationery, Printing and Xerox } \\
\text { Mailing Services and Storage Costs } \\
\text { Telephone and Telegraph } \\
\text { Office Supplies and Services } \\
\text { Staff Travel } \\
\text { Miscellaneous } \\
\text { Administrative } \\
\text { Insurance } \\
\text { Depreciation } \\
\text { Repair and Upkeep-Office Equipment/ }\end{array}$ & $\begin{array}{r}35,735 \\
7,614 \\
19,838 \\
11,190 \\
13,363 \\
4,415 \\
8,746 \\
3,474 \\
2,782 \\
39,601\end{array}$ & $\begin{array}{r}37,000 \\
9,000 \\
20,000 \\
13,000 \\
14,000 \\
5,000 \\
9,000 \\
4,000 \\
5,000 \\
41,000\end{array}$ & $\begin{array}{r}36,408 \\
13,514 \\
10,049 \\
11,467 \\
8,233 \\
4,808 \\
6,800 \\
4,858 \\
3,923 \\
37,229\end{array}$ & $\begin{array}{r}37,000 \\
15,000 \\
12,000 \\
13,000 \\
9,000 \\
5,000 \\
7,000 \\
6,000 \\
4,000 \\
40,000\end{array}$ \\
\hline $\begin{array}{l}\text { Leasing Fees } \\
\text { Annual Leave Expense }\end{array}$ & $\begin{array}{l}18,764 \\
20,984\end{array}$ & $\begin{array}{r}20,000 \\
7,000\end{array}$ & $\begin{array}{l}23,707 \\
10,079\end{array}$ & $\begin{array}{r}25,000 \\
7,000\end{array}$ \\
\hline $\begin{array}{l}\text { TOTAL EQUIPMENT, SUPPLIES AND } \\
\text { ADMINISTRATIVE EXPENSES }\end{array}$ & 186,506 & 184,000 & 171.075 & 180,000 \\
\hline TOTAL GENERAL OPERATING EXPENSES & 647.119 & 661,300 & 644,485 & 736,440 \\
\hline NET IPSA EXPENSE OVER INCOME & - & 28,522 & 33,118 & 0 \\
\hline TOTAL EXPENDITURES & $1,562,252$ & $1,753,166$ & $1,731,251$ & $1,897,206$ \\
\hline
\end{tabular}


Table 6. APSA National Office Salary Scale, 1989-90

\begin{tabular}{|c|c|c|c|c|c|}
\hline \multirow[b]{2}{*}{ APSA Position } & \multirow{2}{*}{$\begin{array}{l}\text { Equivalent } \\
\text { Government } \\
\text { Grade }\end{array}$} & \multirow{2}{*}{$\begin{array}{c}1989 \\
\text { Federal } \\
\text { Salary Scale }\end{array}$} & \multirow{2}{*}{$\begin{array}{c}1989-90 \\
\text { APSA } \\
\text { Salary Scale }\end{array}$} & \multicolumn{2}{|c|}{$\begin{array}{l}\text { Number of } \\
\text { Employees }\end{array}$} \\
\hline & & & & $\begin{array}{l}\text { Full- } \\
\text { Time }\end{array}$ & $\begin{array}{l}\text { Part- } \\
\text { Time }\end{array}$ \\
\hline $\begin{array}{l}\text { Executive Director } \\
\text { Associate Director } \\
\text { Assistant Director } \\
\text { Staff Associate } \\
\text { Senior Administrative }\end{array}$ & $\begin{array}{l}17-18 \\
15-17 \\
13-15 \\
11-14\end{array}$ & $\begin{array}{r}\$ 75,500-86,682 \\
57,158-75,500 \\
41,121-74,303 \\
28,852-63,172\end{array}$ & $\begin{array}{r}\$ 57,448-85,671 \\
48,642-68,200 \\
36,340-56,875 \\
31,997-49,969\end{array}$ & $\begin{array}{l}1 \\
2 \\
0 \\
0\end{array}$ & $\begin{array}{l}0 \\
0 \\
0 \\
2\end{array}$ \\
\hline $\begin{array}{l}\text { Assistant } \\
\text { Administrative }\end{array}$ & $9-10$ & $23,846-34,136$ & $26,76|-36,93|$ & 7 & 0 \\
\hline $\begin{array}{l}\text { Assistant } \\
\text { Secretary } \\
\text { Clerk. Receptionist. }\end{array}$ & $\begin{array}{l}6-8 \\
4-7\end{array}$ & $\begin{array}{l}17,542-28,070 \\
14,067-25,343\end{array}$ & $\begin{array}{l}19,751-29,930 \\
15,855-28,518\end{array}$ & $\begin{array}{l}4 \\
1\end{array}$ & $\begin{array}{l}0 \\
1\end{array}$ \\
\hline Maintenance & $1-3$ & $10,213 \cdot 16,293$ & $11,058-21,436$ & 4 & 0 \\
\hline
\end{tabular}

Table 7. Trust and Development Fund Assets, June 30, 1989

\begin{tabular}{lrrr}
\hline \hline & Total Cost & Total Market Value & \\
\hline Bonds & & & \\
Ford Motor Credit Co. & $\$ 100,750$ & 100,000 & \\
GNMA Pass Thru SF X & 46,259 & 50,974 & \\
U.S. Treasury Note & 50,000 & 26,219 & $12.67 \%$ \\
U.S. Treasury Note & 26,813 & 228,662 & \\
Subtotal & 223,822 & & $8.17 \%$ \\
Bond Mutual Fund & & 147,541 & $15.77 \%$ \\
Northeast Investors Trust & 180,000 & 284,735 & \\
Cash Equivalents & 284,735 & & \\
Equity Mutual Funds & & 122,342 & \\
Fidelity Equity & 85,028 & 114,557 & \\
Gambelli Asset & 115,000 & 82,121 & \\
lvy & 54,029 & 187,670 & 147 \\
Mutual Shares & 90,000 & 195,572 & \\
Rowe T Price & 175,000 & 178,090 & \\
Sequoia & 65,000 & $1,144,499$ & \\
Windsor & 58,911 & $\$ 1,805,437$ & \\
Subtotal & 642,968 & & \\
TOTAL & $\$ 1,331,525$ & & \\
\hline
\end{tabular}




\section{Trust and Development Fund}

The Board of Trustees of the Trust and Development Fund took a cautious approach to the stock market during much of 1988-89, keeping a somewhat larger than normal percentage of assets in cash equivalents. The equity portion of the Trust and Development Fund increased by 15.3 percent during the period from June 30,1988 to June 30,1989 , compared with a gain of 18.9 percent in the S\&P 500 Index. Looked at in another way, the total market value of the Fund's portfolio increased from $\$ 1,703,331$ to $\$ 1,805,439$ over the twelve months ending June 30 , 1989 while the Fund contributed a total of $\$ 184,872$ in dividends and interest to the general operating fund of the Association. The cost of the assets in the portfolio and their market values as of june 30,1989 , are displayed in Table 7.

The Trust and Development Fund contributed 10 percent of the Association's revenue in 1988-89, an important element in its continuing financial health. Under the able guidance of its consultant, Loren Ross, the Trustees of the Fund are committed to continuing a strategy of investment in a diversified set of mutual funds that have a low rate of risk relative to potential return.

\section{Summary}

The Association's financial situation remains sound under the competent direction of Catherine Rudder. Additional revenues have been aggressively pursued and costs have been effectively controlled.

\section{Soviet Political Science Association Hosts APSA Delegation}

Lucian W. Pye

Massachusetts Institute of Technology

As part of a continuing exchange program an APSA delegation-consisting of Pres1- dent Lucian W. Pye, former presidents Richard Fenno, Jr., Samuel P. Huntington, Seymour Martin Lipset, Kenneth Waltz, and professors Alexander Dallin, Gail Lapidus, and Bryon Shafer, and Executive Director Catherine Rudder-visited the Soviet Union from July 2-12, 1989, at the invitation of the Soviet Political Science Association.

In Moscow the roundtable discussions were on the subject of "political reform." Bryon Shafer of Oxford University presented the paper on the American experience. The Soviet participants were exceedingly open and frank in talking about developments with respect to perestroika and glasnost. They were particularly informative about the recent Soviet elections, the rise of legislative authority in the new Supreme Soviets, the prospects of factions in the Communist Party and of multi-party development for the country, and of the nationalities problem in the Soviet Union.

The Americans were impressed with the Soviet enthusiasm for the development of "politology," which is their name for political science. Dr. Georgii Shakhnazarov, president of the Soviet Political Science Association (SPSA) and Central Committee member, called for the "deideologizing" of political analysis and the need for a "value-free and objective" methodology. William Smirnov, the executive director of SPSA, agreed to correspond with Catherine Rudder about future exchange possibilities.

The APSA delegation then briefly visited Leningrad and Tallinn in Estonia. In both of these places meetings were held with not only academicians but also political activists who in the spirit of perestroika had successfully challenged Party candidates in the recent elections. Their enthusiasm for the benefits of political participation contrasted with the more pessimistic outlook of most of the scholars the delegation met. In Tallinn in particular the activists were looking forward to the prospects of "selfmanagement" with great hopes but some lingering fears that an "accident" might end their happiness. None of the Soviet participants at any of the meetings seemed interested in discussing the Chinese experience with "reforms" in the wake of Tiananmen. 